\title{
Modulation of Ca125 expression by hTERT in ovarian cancer : Possible implication of PI3K/akt/mTOR signaling pathway
}

\author{
Atallah D, Antoun S, Moubarak M, El Kassis N, Chahine G, Hilal G \\ Hôtel-Dieu de France University Hospital, Saint Joseph University, Beirut, Lebanon
}

\section{AlM}

The aim of our study was to investigate the possible inter-relationship between Ca125 secretion and telomerase activity, and the possible implication of the PI3K/Akt/mTOR pathway in this modulation. In addition, the effect of the neoadjuvant chemotherapy on both hTERT and Ca125 expression was studied.

\section{METHODS}

- Ovarian cancer cell lines OVCAR-3, SK-OV-3 and IGROV-1 were treated with three different telomerase inhibitors, BIBR-1532, Costunolide and MST-312, one activator, LPS, and various inhibitors of the PI3K/Akt/mTOR pathway, PI828, wortmanin, GSK692690 and rapamycin.

- The three cell lines were treated with 20 uM Cisplatin and $100 \mathrm{nM}$ Paclitaxel for 48 hours. Sk-Ov-3 and Igrov-1 were treated with the same concentration of cisplatin once per week for one hour and of paclitaxel once per week for three hours during 6 weeks.

- Effect of telomerase siRNA transfection on Ca125 production was tested by Transfection with siRNA of hTERT subunit.

- The assessment of Ca125 and hTERT mRNA expression was performed using qPCR technique.

\section{RESULTS}

- The three telomerase inhibitors, costunolide and BIBR 1532 at 5 and $10 \mathrm{mM}$ and MST-312 at 1 and $2 \mathrm{mM}$, decreased the Ca125 mRNA expression and protein secretion by the three cell lines. The same pattern was obtained when cells were treated with $h T E R T$ siRNA.

- The activation of hTERT lead to an increase in Ca125 expression and secretion, and to an increase in cell migration and motility.

- Interestingly, inhibition of PI3K/Akt/mTOR signaling pathway by PI828, wortmanin, GSK692690 and rapamycin lead to a decrease in Ca125 concentration suggesting the involvement of this pathway in Ca125 regulation.

- Moreover, an additive effect was shown when costunolide and BIBR 1532 were combined with the previous inhibitors. A decrease in telomerase expression and activity was obtained after gene silencing of $\mathrm{Ca} 125$ by the three cell lines, along with a decrease in PI3K, Akt and mTOR gene expression, which may explain the possible implication of this signaling pathway in the modulation of hTERT by Ca125.
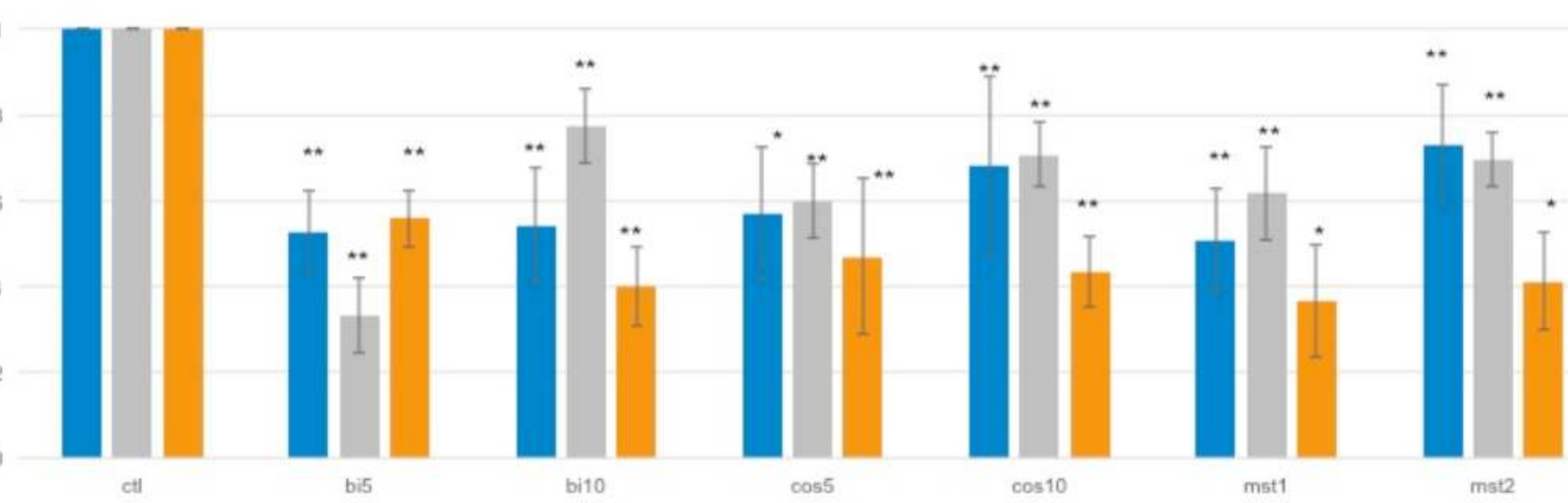

Figure I: Effect of hTERT inhibitors on the three cell lines

$$
\text { Effect of pathway signaling inhibitors on Ca125 }
$$
mRNA expression in cells

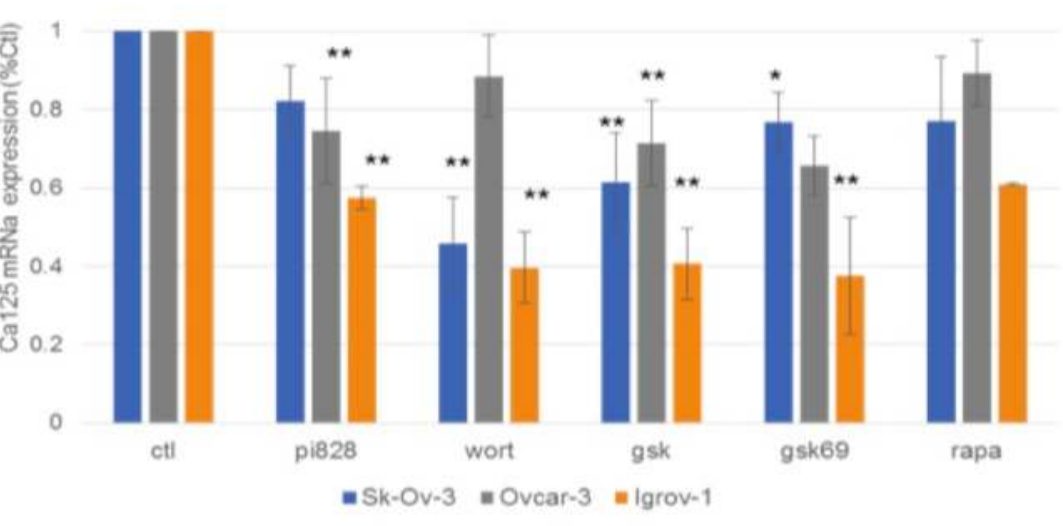

Figure 2: Effect of pathway inhibitors on Cal 25 expression

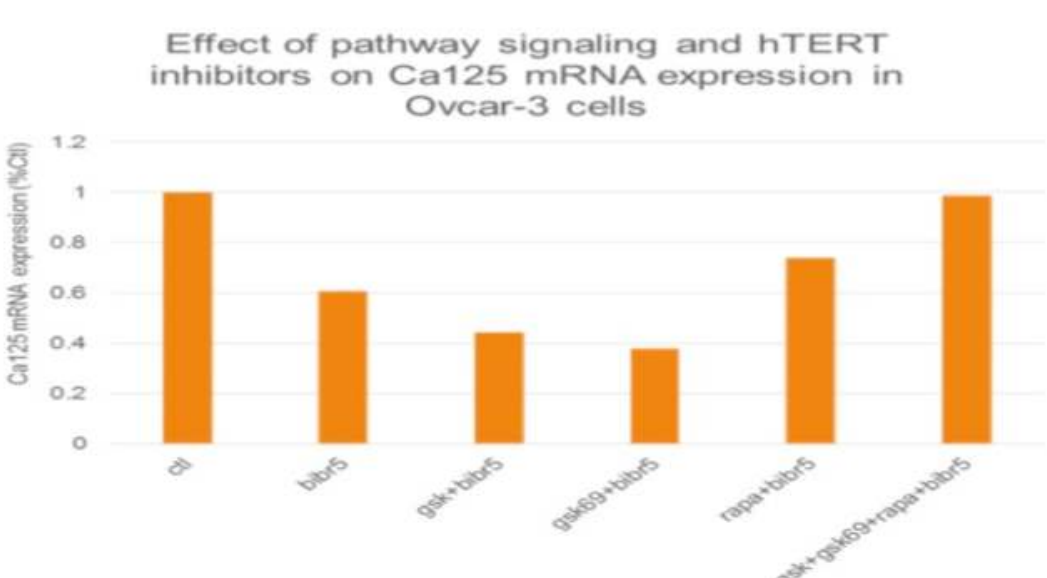

Figure 3: Effect of pathway signaling and hTERT inhibitors on Cal25 mRNA expression

\section{CONCLUSION}

Both inhibition of telomerase and PI3K/Akt/mTOR signaling pathway decreased the Ca125 secretion, while inhibition of Ca125 decreased hTERT expression and activity suggesting a mutual modulation and a substantial role of Ca125 in cancer initiation and progression 